\title{
Ethnographie des transmissions familiales de la langue bretonne en Basse-Bretagne
}

Ethnography of Breton language transmission in families in Lower Brittany

\section{Gaëlle Violo}

\section{Q OpenEdition}

\section{Journals}

Édition électronique

URL : https://journals.openedition.org/lbl/1518

DOl : $10.4000 / \mathrm{lbl} .1518$

ISSN : 2727-9383

Éditeur

Université de Bretagne Occidentale - UBO

\section{Édition imprimée}

Date de publication : 1 novembre 2011

Pagination : 7-18

ISBN : 978-2-901737-92-6

ISSN : $1270-2412$

Référence électronique

Gaëlle Violo, «Ethnographie des transmissions familiales de la langue bretonne en Basse-Bretagne », La Bretagne Linguistique [En ligne], 16 | 2011, mis en ligne le 01 mai 2021, consulté le 22 mai 2021. URL : http://journals.openedition.org//bl/1518; DOI : https://doi.org/10.4000//bl.1518

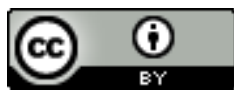

La Bretagne Linguistique est mise à disposition selon les termes de la Licence Creative Commons Attribution 4.0 International. 
Gaëlle VIOLO*

\title{
Ethnographie des transmissions familiales de la langue bretonne en Basse-Bretagne
}

\begin{abstract}
L
a transmission est une notion centrale en anthropologie. Dans le cadre de ma recherche ${ }^{1}$, j'ai choisi de l'étudier à travers la pratique linguistique. Comment se transmet une langue dans la famille? Avec qui? Où? Pourquoi? Les réponses à ces questions sont d'autant plus complexes lorsqu'elles s'appliquent aux locuteurs des langues dites «minoritaires», «régionales».

Ma thèse s'inscrit dans une démarche ethnologique, se caractérisant par un lieu, une démarche (un point de vue, un éclairage), une méthode spécifique, et une finalité analytique ${ }^{2}$.

J'ai opté pour deux lieux. Mon étude porte à la fois sur les transmissions familiales de la langue bretonne, en Basse-Bretagne, et sur celles de la langue française, en Saskatchewan, au Canada. Quelles sont les logiques sous-tendant la transmission familiale d'une langue, moins parlée, aujourd'hui, dans les sphères publique et privée?
\end{abstract}

* Doctorante en ethnologie, CRBC, UBO/ueb

1. Cette recherche doctorale a été financée par la Région Bretagne pour une durée de trois ans, et menée au sein du Centre de Recherche Bretonne et Celtique tout en étant rattachée à l'Université Européenne de Bretagne (UEB/UBO).

2. Mondher KILANI, Introduction à l'anthropologie, Lausanne, Payot Lausanne, 1996 (1992), p. 33-34. 
J'ai mené un premier terrain en Basse-Bretagne, où le nombre de locuteurs premiers du breton est en nette diminution depuis la seconde moitié du XX $\mathrm{X}^{\mathrm{e}}$ siècle. Un second a été entrepris en Saskatchewan, province anglophone du centre-ouest du Canada, où vivent des personnes ayant le français comme première langue. L'histoire du peuplement du pays explique ce phénomène. Là-bas, les locuteurs du français sont moins nombreux que ceux de l'anglais.

Ces deux enquêtes donnent un éclairage (réciproque) sur deux réalités appartenant à un même monde globalisé. D'une part, ce type de comparaison permet d'examiner et de saisir « les logiques locales qui se mettent en place en rapport avec les logiques globales de plus en plus envahissantes ${ }^{3} \gg$. D'autre part, il semble intéressant de placer en parallèle deux situations où le français n'est pas toujours «un voisin envahissant ${ }^{4} \gg$.

Ma méthode, ethnologique, a permis de recueillir la parole des individus, locuteurs (ou non) des langues concernées. Il m'a fallu concilier à la fois le point de vue extérieur du chercheur, qui se veut le plus objectif possible, et celui de l'acteur - de l'observé, de l'interlocuteur, de l'informateur - pour comprendre les mécanismes de l'intérieur. L'ethnologue doit, en effet, être en mesure de relever des indicateurs qui lui serviront à élaborer son propre regard. Par conséquent, sa tâche est aussi de capter les marqueurs des informateurs, autrement dit ce qui fait sens pour la population qu'il interroge.

J'ai conduit le terrain en Bretagne, de 2007 à 2009, principalement en réalisant une quarantaine d'entretiens. Les personnes interrogées appartenaient toutes à des familles dans lesquelles, historiquement, le breton est, était, parlé. J'ai rencontré des locuteurs du breton - des grands-parents, des parents, des enfants -, et des nonlocuteurs. J'ai tenu d'autres entretiens avec les personnes en charge des politiques linguistiques à la Région Bretagne, ainsi que celles missionnées en faveur de la pratique du breton au sein du Conseil Général du Finistère ${ }^{5}$.

3. Mondher KILANI, op. cit., 1996 (1992), p. 300.

4. Jean LE Dû, «Un voisin envahissant : le français dans la langue bretonne», dans Regards étonnés, de l'expression de l'altérité à la construction de l'identité, Brest, Les Amis de Gaël Milin, 2003, p. 503-512.

5. Ces entretiens ont été menés avant les élections régionales du printemps 2010. De 
Mes objectifs dans cet article sont de présenter ma façon d'aborder les transmissions familiales de la langue bretonne, et de partager mes premières analyses. L'étude au Canada, Saskatchewan, n'est pas relatée dans cet écrit.

J'introduirai la notion de trajectoire biographique me permettant d'aborder les transferts linguistiques intergénérationnels. Je l'illustrerai ensuite par un cas concret, celui de Marie, tiré de mon expérience de terrain en Bretagne. Puis, je proposerai une lecture analytique de la transmission à travers la dynamique «donner, recevoir, rendre ». Enfin, grâce à mon travail ethnographique et à l'examen des logiques présentes dans les transmissions familiales, j'en suggérerai une classification.

\section{La transmission et la trajectoire biographique de l'objet}

L'idée souvent véhiculée par la transmission est celle d'une continuité entre les générations, et d'une reproduction fidèle de l'objet (matériel ou immatériel). La transmission adopte des parcours sinueux. Elle n'est pas seulement l'affaire des plus âgés dont la tâche serait de passer un objet à la génération suivante. Les plus jeunes jouent un rôle dans le mécanisme de passation intergénérationnelle. Ils peuvent même le déclencher lorsque leur regard se porte derrière eux, sur le vécu familial notamment. J'envisage la transmission comme un mécanisme fait de sélections, de choix. Par définition, des objets sont laissés pour mieux en transmettre d'autres. La transmission, tout en donnant une impression de continuité, est parcourue de discontinuités, voire de ruptures. Nombre d'études ont d'ailleurs «remis en cause l'idée d'une totalité sociale et culturelle qui se reproduirait à l'identique de génération en génération ${ }^{6} \gg$.

La difficulté, dans l'étude d'une transmission, réside dans son mécanisme, son processus impalpable qu'il faut saisir au vol. Parfois, malgré de nombreuses observations et entretiens, elle paraît encore

nouvelles rencontres seront organisées avec les personnes occupant désormais les postes concernés.

6. Catherine ChORON-BAIX, «Transmettre et perpétuer aujourd'hui», Ethnologie Française, n $^{\circ} 30,2000$, p. 357-360, p. 357. 
fugace. Denis Chevallier et Isac Chiva titraient l'introduction de l'ouvrage Savoir-faire et pouvoir transmettre ${ }^{7}$, "L'introuvable objet de la transmission ${ }^{8}$. Ils y décrivaient les difficultés de description et d'analyse :

«Comment, face à des manières de faire si diverses et complexes, isoler les modèles et comprendre les mécanismes de leur transfert et de leur acquisition, alors que ni les uns ni les autres ne sont explicitables ou explicités par les intéressés euxmêmes ${ }^{9}$ ?»

Les informateurs sont en effet rarement en mesure de produire un discours qui expliciterait les processus de transfert entre individus, entre générations. Lorsqu'il s'agit d'évoquer la transmission d'une langue, les personnes la décrivent souvent de manière vague : «Je ne leur ai pas parlé», «Je l'ai appris», «Je l'ai entendu chez les voisins». En effet,

«la pratique linguistique est difficilement mesurable et sa transmission l'est d'autant plus qu'il existe des degrés différents de transmission et de réception; il s'agit avant tout d'un processus qui se construit dans l'interaction et reste nécessairement évolutif, ce qui ne signifie pas que cette transmission ne peut, à un moment précis, être évaluée ${ }^{10}$.»

Dans le cadre de ma recherche, j'ai souligné que l'un des objectifs était de décrire, de comprendre les modes de transmission de la langue bretonne en Basse-Bretagne (France) d'abord, et de la langue française en Saskatchewan (Canada) ensuite. Dans les deux cas, la transmission n'est pas évidente : elle n'est plus systématique, automatique. Une hétérogénéité apparaît clairement selon les familles.

7. Denis Chevallier, Isac Chiva éd., Savoir-faire et pouvoir transmettre, Paris, Maison des Sciences de l'Homme (Collection Ethnologie de la France, cahier 6), 1996 (1991).

8. Denis Chevallier, Isac Chiva, «Introduction. L'introuvable objet de la transmission », dans D. Chevallier, I. ChIVA éd., op. cit., 1996 (1991), p. 1-12.

9. Denis Chevallier, Isac Chiva, op. cit., 1996 (1991), p. 9.

10. Alexandra FILHON, Langues d'ici et d'ailleurs : transmettre l'arabe et le berbère en France, Paris, INED, 2009, p. 99. 
Pour bien saisir les rouages du transfert linguistique, j'ai convoqué plusieurs bases théoriques.

Dans un premier temps, l'hypothèse était qu'à travers une histoire de vie, une histoire familiale, il était possible d'appréhender la (non)transmission de la langue. Les choix, les contextes seraient alors mis en lumière. Christine Deprez, dans une approche qu'elle nomme "(auto)biographique», et qui s'inscrit dans le champ de la sociolinguistique met en avant l'importance du discours que les personnes tiennent sur la langue et sa pratique ${ }^{11}$. À travers le parcours de l'individu et de sa famille, la trajectoire de l'objet-langue prend place.

Alexandra Filhon traite, elle, de la transmission de l'arabe et du berbère dans des familles avant leur migration de leur pays d'origine et après leur établissement en France. Elle suit une démarche sociologique et met en évidence l'importance de la prise en compte des parcours de vie, marqués par la migration d'un pays à un autre. L'idée est que

«les transmissions familiales sont diverses, perceptibles ou non, souhaitées ou pas. [...] Si l'origine sociale des individus est un déterminant central dans la compréhension des héritages familiaux, seule l'analyse des parcours des individus permet d'appréhender plus finement les mécanismes de transmission ou de non-transmission ${ }^{12} . »$

Enfin, il m'a semblé opportun de prendre modèle sur des travaux ethnologiques déjà réalisés sur la transmission des objets ou des techniques, et notamment celui de Thierry Bonnot, La vie des objets $^{13}$, inspiré par les écrits de Igor Kopytoff ${ }^{14}$. Dans cette étude, il décrit l'itinéraire biographique des poteries de grès, "ensemble

11. Christine Deprez, «Histoires de langues, histoires de vies, Modes d'expression de l'identité linguistique dans les histoires de vie des personnes bilingues», Cahiers de Sociolinguistique (PUR), n 5, 2000, p. 167-174, p. 167.

12. Alexandra FILHON, op. cit., 2009, p. 17-18.

13. Thierry Bonnot, La vie des objets, Paris, Maison des sciences de l'homme (Collection Ethnologie de la France), 2002.

14. Igor KopytofF, "The cultural Biography of Things: Commoditization as Process", in A. Appadurai éd., The Social Life of Things: Commodities in Cultural Perspective, Cambridge, Cambridge University Press, 1986, p. 64-94. 
d'objets a priori banals», produits dans des usines de Saône-etLoire (désormais fermées), et "considérés aujourd'hui comme partie intégrante du patrimoine local ${ }^{15} \gg$. Son objectif est alors d'amener

«l'anthropologie à considérer l'objet dans sa trajectoire globale, de sa production à sa consommation, en passant par l'échange et sa distribution. [...] Tenter de saisir la biographie d'une chose équivaut donc à étudier l'histoire de ses singularisations successives, et des classifications et reclassements qu'elle subit selon les catégories socialement construites ${ }^{16}$.»

Les notions de parcours, de trajectoire et d'itinéraire semblent de bons outils pour retracer la place de l'idiome dans les familles, comprendre ses changements de statuts (langue du quotidien, langue connue sans être utilisée, langue oubliée, langue patrimoniale?) au sein des foyers. Ceci permet également de mieux saisir les différents sens donnés, à l'heure actuelle, à la langue bretonne ici et au français là-bas.

\section{Ethnographie : le cas de Marie}

Je retracerai maintenant la trajectoire biographique du breton dans la famille de Marie, afin d'illustrer la complexité du processus de transmission familiale.

Marie, âgée d'une cinquantaine d'années, vit dans le pays d'Auray. «J'ai un peu entendu mes parents parler breton quand ils voulaient pas qu'on comprenne», dit-elle au début de la conversation. Elle m'explique que ses parents parlent breton, mais ne l'ont pas appris dans leur famille. Son père l'a davantage attrapé avec le voisinage et dans les bois (il est devenu ensuite menuisier). Sa mère l'a appris au catéchisme, même si cela paraît «paradoxal» dit-elle, et dans le bistrot familial. D'après Marie, ses parents jugent le breton inutile. Ses deux sœurs ne le connaissent pas.

Marie s'est initiée au breton, au lycée, en option sur l'heure des repas. Puis elle a suivi des cours du soir. Petit à petit, elle a fini par passer une licence de breton, par correspondance, à Rennes. Dès ce

15. Thierry BonNOT, op. cit., 2002, p. 5.

16. Thierry BonNOT, op. cit., 2002, p. 5. 
début d'apprentissage, elle est allée parler breton avec sa grand-mère (et «ses copines»), avec laquelle elle a bavardé dans cette langue pendant vingt ans, jusqu'à la mort de l'aïeule.

Marie maitrise couramment le breton. Elle enregistre aujourd'hui quelques émissions en langue bretonne sur la radio locale et intervient dans les écoles.

Marc, son mari, est issu d'une famille où le breton est connu, et a lui aussi fait la démarche de l'apprendre. «On ne s'est pas connu en breton, du coup on n'a jamais réussi à s'y mettre après ensemble. C'est-à-dire que s'il y a une tierce personne on parle breton, mais tous les deux non...». Ils ont six enfants.

Tous deux ont choisi d'introduire le breton à la maison. Marie, plus que son époux, l'a mis en pratique avec ses enfants. À la naissance de leur première fille, elle ne se sentait pas tout à fait capable de lui transmettre. Elle trouve cela stupide aujourd'hui. Ils ont donc scolarisé leurs trois premiers enfants, à temps partiel, dans une école Diwan. Mais, son «désir» était de faire l'école à la maison. Finalement, pour leurs six enfants, elle a majoritairement pris en charge, à la maison et en breton, l'enseignement maternel et primaire. Au collège, puis au lycée, ils ont tous intégré des établissements Diwan.

Chez eux, ils emploient le français et le breton, selon les envies des uns et des autres. Il n'y a pas d'obligation.

Les enfants, plus jeunes, ont pu parler avec leur arrière grandmère. En revanche, Marie, Marc, et les enfants communiquent en français avec les grands-parents, puisqu'ils ne souhaitent pas le faire en breton. Marie disait :

«Disons que quand ils sont très en forme, ils sortent deux ou trois petits trucs, surtout que mon père connaît énormément de choses, des phrases que je note tout de suite [...]. Les enfants ont essayé de leur parler, mais je pensais qu'ils auraient parlé aux enfants, mais là non plus ça n'a pas marché avec mes enfants. Parce qu'en fait moi j'ai parlé avec ma grand-mère. J'ai eu cette chance-là, d'ailleurs elle qui n'a pas transmis le breton à ses enfants, à ses filles.»

En m'évoquant la non-communication en breton entre ses parents et ses enfants, Marie précise : «Je ne désespère pas que cela se fasse un jour», autrement dit que son père utilise le breton avec 
ses petits-enfants, comme elle a pu le faire avec sa propre grandmère. Elle argumente, en remarquant qu'il s'intéresse beaucoup à ses émissions radiophoniques, à ses interventions en breton... Elle note aussi que, paradoxalement, il est assez fier qu'elle pratique le breton. Marie raconte qu'un jour, alors qu'il discutait avec quelqu'un, il la présenta en ces termes : "Ah bah c'est ma fille, et puis vous savez, elle parle breton !!»

\section{Donner, recevoir, rendre}

Désormais, parler breton à ses enfants dès leur plus jeune âge n'est plus une évidence. Le faire est une démarche qui suscite réflexion et volonté.

Marcel Mauss, qualifié souvent comme le fondateur de l'anthropologie française, a mis en évidence, dans Essai sur le don ${ }^{17}$, que l'échange était réparti en trois obligations : l'obligation de donner, de recevoir et de rendre. Une invitation, par exemple, doit être acceptée, donc reçue, mais aussi, de manière implicite, elle devra être rendue, pour que les relations soient maintenues. Il montre ainsi que ces échanges permettent aux individus, aux groupes, de maintenir des liens entre eux. Marcel Mauss les qualifie de «faits sociaux totaux», «c'est-à-dire qu'ils mettent en branle dans certains cas la totalité de la société et de ses institutions [...], et dans d'autres cas, seulement un très grand nombre d'institutions, en particulier lorsque ces échanges et ces contrats concernent plutôt des individus ${ }^{18}$ ». L'essai a beaucoup été repris et réinterprété, et notamment dans le domaine de la famille, dans les analyses des modes de transmissions intergénérationnelles.

Françoise Bloch et Monique Buisson, dans un article intitulé «La circulation du don entre générations, ou comment reçoit-on?», pensent le lien familial comme une articulation entre le don et la dette. Elles exposent, en effet, que les rapports entre donataires et donateurs sont intimement liés aux capacités de chacun à recevoir, acte qui détermine de facto l'aptitude pour donner à son tour. En cas

17. Marcel MAUSS, «Essai sur le don. Forme et raison de l'échange dans les sociétés archaïques», dans Sociologie et Anthropologie, Paris, PUF (Quadrige), 2006 (1924), p. 145-279.

18. Marcel MAUSS, op. cit., 2006 (1924), p. 274. 
de non réception, il est alors impossible de (re)donner et une dette s'instaure ${ }^{19}$.

Anne Gotman traite plus spécifiquement de l'héritage. Elle explique que la réception de l'objet hérité est primordiale pour que celuici soit éventuellement redonné à la génération suivante ${ }^{20}$. En étudiant les phénomènes de dilapidation de l'héritage, l'auteure montre que le point crucial dans ce processus est celui de réception. Pour souscrire à l'héritage, il faut accepter de se positionner dans la lignée familiale, et «se reconnaître fils de... ${ }^{21} »$. Si l'individu est d'accord pour le recevoir, celui-ci pourra être, par la suite, passé (et parfois enrichi) à la génération suivante. La séquence réception/appropriation est le temps décisif du mode de transmission par héritage.

À travers la description de la transmission du breton dans la filiation de Marie, je note que les points déterminants sont également liés à l'acte de réception de la langue. Si une transmission y est possible aujourd'hui, c'est que celle-ci a été activée par une volonté de recevoir la langue. Marie, ressentant un manque, a voulu apprendre le breton, en prenant des cours et en demandant à sa grand-mère de lui parler. Cette aïeule a accepté de donner ce qu'elle n'avait pas passé à ses filles. Ses sœurs, quant à elles, ne souhaitent pas recevoir la langue. La connaissance du breton, de leur côté, s'interrompt, pour l'instant.

Les parents de Marie ont comme une dette vis-à-vis d'elle en refusant, encore aujourd'hui, de transmettre leur breton.

Marie et Marc ont passé le breton à leurs enfants. La capacité et la volonté de ces derniers de communiquer dans cette langue avec leur progéniture dépendra très certainement de la manière dont ils ont reçu et se sont appropriés la langue. Ceci sera conditionné, en grande partie, par leur positionnement face à la filiation et à leur héritage parental.

19. Françoise Bloch, Monique BuIsson, «La circulation du don entre générations, ou comment reçoit-on?», Communications, n 59, 1994, p. 55-72.

20. Anne Gotman, Hériter, Paris, PUF, 1988, et «L'individu saisi par l'héritage», dans F. Singly (de), S. Mesure éd., Le lien social, Paris, PUF, 2001, p. 223238.

21. Anne Gotman, op. cit., 2001, p. 225. 
Le processus de transmission n'est donc pas à envisager dans une logique descendante, des parents aux enfants, mais bien l'inverse. Mieux vaut la concevoir comme une dynamique allant du présent vers le passé et marquée par cette volonté des uns d'avoir un bien que leurs prédécesseurs ne leur ont pas donné.

\section{DES transmissions : UNE classification}

Par l'itinéraire biographique de la langue dans les familles, et par la mise en évidence de la réception/appropriation comme point clef, j'ai pu établir une classification des différents modes de transmission. Elle est une manière d'ordonner le réel, et n'est en rien figée. Elle est à replacer à un moment donné, celui de la recherche.

Les critères retenus sont uniquement basés sur des cas que j'ai pu rencontrer. Je ne mentionne pas des situations pouvant exister, mais que je n'ai pas croisées et interrogées au cours du terrain. Quatre types de transmission ont été dégagés dans le cas breton. Dans cette typologie, trois générations sont d'emblée prises en compte : celle des grands-parents (60/80 ans), celle des enfants devenus parents $(35 / 60)$, et celle des petits-enfants (moins de 35 ans).

\section{Le premier est celui d'une interruption de la transmission.}

Le breton, langue d'usage dans la famille, devient une langue d'adulte non parlée aux enfants, et donc non transmise. Le breton n'est plus utilisé au profit du français. Une ou deux générations sont en mesure de le parler : les plus anciennes. Les autres, au mieux, l'ont «dans l'oreille». Dans la génération qui ne le connaît pas, personne ne fait la démarche de l'apprendre en dehors de la famille, même si elle regrette de ne pas le connaître, ou s'y intéresse.

Le deuxième est celui d'un hiatus de la transmission.

Un hiatus est un «espace entre deux choses, dans une chose ${ }^{22}$ ». Il semble que ce terme soit approprié pour désigner le fait que la transmission de la langue bretonne se soit arrêtée pendant un temps donné. Une génération ne l'ayant pas reçue comme langue première décide de l'apprendre. Malgré cela, il n'y a pas d'échanges entre les apprenants et les locuteurs natifs d'une même famille. La langue est

22. Le Nouveau Petit Robert, Paris, Dictionnaires Le Robert, 1995. 
donc connue par plusieurs générations de la filiation, sans que cela puisse créer un lien spécifique. La dette n'est pas comblée.

Le troisième cas est celui d'une halte de la transmission intergénérationnelle.

Une halte se définit comme étant, au sens figuré, "un moment de pause, une interruption momentanée au cours d'une action ou d'une évolution ${ }^{23} \gg$. Dans mon étude, cette halte se caractérise par un arrêt de la transmission du breton, donc de son usage, des parents aux enfants. Il est suivi, plusieurs années après, par un apprentissage du breton d'une ou des générations ne l'ayant pas reçu dans la famille.

Cet apprentissage permettra de réintroduire le breton dans les échanges entre les générations, à la fois entre celles qui l'ont comme langue première et les autres, et de rétablir une transmission, partielle ou complète, avec les générations suivantes. La circulation de la langue se réamorce. C'est le cas de Marie avec sa grand-mère.

Enfin, le dernier cas est celui du regain de transmission : le regain étant «le retour de... ce qui était compromis, ce qui avait disparu ${ }^{24} »$. Un retour vers la langue bretonne s'opère grâce à un apprentissage ou à la scolarisation des enfants dans des classes bilingues... Par exemple, le breton a cessé d'être parlé, notamment par les grands-parents actuels et leurs petits-enfants peuvent être scolarisés dans des écoles bilingues français-breton ou dans des filières d'immersion. Les parents, eux, ne connaissent et ne comprennent pas le breton. Il n'est pas inséré dans la vie de famille et reste une connaissance sans pratique dans la parenté. L'appropriation de la langue par les enfants est ici importante pour que le breton bascule éventuellement du statut de langue scolaire à celui de langue familiale et sociale.

\section{Conclusion}

J'ai suivi les parcours sinueux des transmissions familiales. J'ai tenté de saisir les moments d'interruption, les haltes et hiatus, les regains dans les transferts linguistiques. J'ai cherché à comprendre les motivations individuelles et familiales à parler breton aujourd'hui.

23. Le Nouveau Petit Robert, op. cit., 1995.

24. Le Nouveau Petit Robert, op. cit., 1995. 
Comme nombre de transmissions, celle du breton n'est ni continue, ni linéaire, ni même seulement descendante. Elle est rythmée par des ruptures plus ou moins franches qui suscitent un désir de réception chez certains. Autant d'analyses et d'observations qui structurent mon regard d'ethnologue sur une pratique. Elles permettent également de réfléchir sur l'évolution de la place de l'idiome dans la société. Langue du passé, du présent, de l'avenir? Dès lors, il est intéressant de relever l'inscription à la Constitution française, en juillet 2008, de l'article 75-1. Il stipule : «Les langues régionales appartiennent au patrimoine de la France ${ }^{25}$.»

Il me semble que l'étude des transmissions familiales de la langue bretonne m'autorise à soulever la question suivante : est-elle un patrimoine comme la Constitution le suggère? Il faudra donc, sans doute, méditer les propos de Jean Pouillon :

«Laisser tomber une part de l'héritage, c'est choisir consciemment ou inconsciemment d'en maintenir une autre, car les sociétés qui se disent modernes ne sont pas des sociétés qui se défont de leur passé : elles le réaménagent en fonction de leurs besoins présents. Comme, en outre, elles sont complexes et conflictuelles, plusieurs types de réaménagement sont en concurrence, et des contemporains s'affrontent par passés interposés en se choisissant leurs ancêtres $[\ldots]$ : ce sont les fils qui engendrent leurs pères pour justifier les changements réels qu'ils apportent au système existant ${ }^{26} . »$

Restera alors, dans un temps futur, à évaluer les «réaménagements» que les acteurs auront effectués, ainsi que leurs impacts.

25. Constitution de la République française, Constitution du 4 octobre 1958, Article 75-1 introduit par l'article 40 de la loi constitutionnelle ${ }^{\circ} 2008-724 \mathrm{du}$ 23 juillet 2008.

26. Jean Pouillon, «Plus c'est la même chose, plus ça change», dans Le cru et le $s u$, Paris, Seuil, 1993, p. 79-91, p. 86-87. 